Article

\title{
Dynamic Intuitionistic Fuzzy Multi-Attribute Group Decision-Making Based on Power Geometric Weighted Average Operator and Prediction Model
}

\author{
Kedong Yin ${ }^{1,2,3}$, Pengyu Wang ${ }^{1, *}$ and Xue Jin ${ }^{1,2} \mathbb{D}$ \\ 1 School of Economics, Ocean University of China, Qingdao 266100, China; \\ yinkedong@126.com (K.Y.); jinxue@ouc.edu.cn (X.J.) \\ 2 College of Oceanic and Atmospheric Sciences, Ocean University of China, Qingdao 266100, China \\ 3 Ocean Development Research Institute, Major Research Base of Humanities and Social Sciences of \\ Ministry of Education, Ocean University of China, Qingdao 266100, China \\ * Correspondence: wangpengyu@stu.ouc.edu.cn; Tel.: +86-155-6322-2277
}

Received: 10 October 2018; Accepted: 18 October 2018; Published: 23 October 2018

\begin{abstract}
With respect to dynamic multi-attribute group decision-making (DMAGDM) problems, where attribute values take the form of intuitionistic fuzzy values (IFVs) and the weights (including expert, attribute and time weights) are unknown, the dynamic intuitionistic fuzzy power geometric weighted average (DIFPGWA) operator and the improved IFVs' GM(1,1) prediction model (IFVs-GM(1,1)-PM) are proposed. First, the concept of IFVs, the operational rules, the distance between IFVs, and the comparing method of IFVs are defined. Second, the DIFPGWA operator and the improved IFVs-GM(1,1)-PM are defined in detail. Third, corresponding decision-making (D-M) steps are proposed. Three kinds of weights are given by the proposed determination method. Finally, an example is given to prove the effectiveness and superiority of the proposed decision-making method.
\end{abstract}

Keywords: the dynamic intuitionistic fuzzy power geometric weighted average (DIFPGWA) operator; IFVs GM(1,1) prediction model; dynamic intuitionistic fuzzy multi-attribute group decision-making

\section{Introduction}

Decision-making is an activity frequently occurring in management. This is the basic idea of modern decision theory. One of the important components in modern decision theory is multi-attribute group decision-making (MAGDM). This refers to the decision-making problem of selecting the optimal alternative or scheduling the scenario when multiple decision makers consider multiple attributes. However, many things in real life have fuzziness and uncertainty, and there is no clear statement. In the description of fuzziness and uncertainty, intuitionistic fuzzy sets have unparalleled advantages. If intuitionistic fuzzy sets are applied to MAGDM and generalized to multi-period decision-making, this decision-making process is called dynamic intuitionistic fuzzy multi-attribute group decision-making (DIF-MAGDM).

Since the Bulgarian scholar Atanassov [1] proposed the concept of intuitionistic fuzzy sets (IFSs), the research on the theory of IFSs has come a long way. Intuitionistic fuzzy sets have been widely used in many real-world cases [2-5]. Many scholars have proposed many improvements to MAGDM and multiple criteria decision making (MCDM) on IFS's methods and models. For example, Liu et al. [6] introduced intuitionistic trapezoidal fuzzy number and proposed an intuitionistic trapezoidal fuzzy prioritized ordered weighted aggregation (ITFPOWA) operator; Hashemi et al. [7] combined ELECTRE (originated in Europe in the mid-1960s. The acronym ELECTRE stands for: ELimination Et Choix Traduisant la REalité (ELimination and Choice Expressing REality)), VIKOR (VIseKriterijumska Optimizacija I Kompromisno Resenje, that means: Multicriteria Optimization and Compromise 
Solution, with pronunciation: vikor) and GRA (grey relational analysis) theory to propose a new decision model based on IFS; Kahraman et al. [8] proposed an EDAS-based (based on the distance from Average Solution) MCDM method and gave the corresponding sensitivity analysis. In many decision-making methods, it is a very important method to solve problems by using operators. Many operators were proposed based on IFSs, such as an intuitionistic fuzzy ordered weighted averaging (IFOWA) operator, intuitionistic fuzzy weighted averaging (IFWA) operator, intuitionistic fuzzy weighted geometric (IFWG) operator, and intuitionistic fuzzy ordered weighted geometric (IFOWG) operator [9-11]. Although the calculation of the above operator is simple, some facts are ignored. However, these operators ignore a practical problem, that is, decision-makers will have their own preferences, with their own subjectivity. Therefore, Tan and Chen [12] proposed an IF choquet integral operator that fully considers the interaction between decision maker preferences. There are still some problems that have not been taken into consideration. That is, the default alternatives and attributes are both independent. In fact, there is a certain link between them. Xu [13] proposed the IFPWA operators and IFPWG operators, which can capture the delicate nuances of the comprehensive evaluation values that the decision makers need to reflect when evaluating. In addition, IFSs are also combined with grey relational models [14], TOPSIS (Technique for Order Preference by Similarity to an Ideal Solution) methods [15], prospect theories [16], evidential reasoning theories [17], and Frank t-norms family [18], and an improved IF-MADM (multi-attribute decision-making) based on the above theory is constructed, further enriching the IFS theory. However, the above method for IF-MADM only addresses static evaluation information at a certain period. In fact, the evaluation of things at one point in time is one-sided. Things evolve over time, and static evaluation cannot reflect the development trend of things. For DIF-MADM, $\mathrm{Xu}$ and Yager [19] proposed a dynamic intuitionistic fuzzy weighted averaging (DIFWA) operator and uncertain dynamic intuitionistic fuzzy weighted averaging (UDIFWA) operator. Wei [20] proposed the DIFWG operator and UDIFWG operator. Su et al. [21] proposed a method that combines TOPSIS method with DIFWA operator and HWA operator to solve DIF-MAGDM. Park et al. [22] extends the VIKOR method and combines (DIFWG) operator and (UDIFWG) operator to solve DIF-MAGDM. Chen et al. [23] proposes a dynamic IF compromise decision method based on time degree. Although the above method solves DIF-MAGDM to some extent from different perspectives, none of them considers the relationship between attribute values. In fact, there are certain links between attributes, alternatives, and periods. Therefore, dynamic intuitionistic fuzzy power geometric weighted average (DIFPGWA) operator that better reflect support relationship between attributes are proposed, which can capture the delicate nuances of the comprehensive evaluation values that the decision makers need to reflect when evaluating. However, it is inaccurate to make judgments by merely aggregating evaluation information from past to present. This may happen because the evaluation of the alternative one by the experts started poorly and then gradually got better. The evaluation of the alternative two started better and then slowly deteriorated. This situation may get the same result through DIFPGWA operator aggregation information. However, this method also has some shortcomings. Therefore, in order to compensate for the lack of DIFPGWA operator, the GM(1,1)-PM based on IFVs that were proposed by Li et al. [24] have been improved. Comprehensive evaluation values of each alternatives could be predicted in the next period, and then make more accurate judgments.

Weight is a relative concept. It is very important to determine the weight in DIF-MAGDM. In this article, attribute weights, expert weights, and time weights are all unknown. For expert weights, Chen and Yang [25] proposed a method for deriving expert weights from evaluation values. Because each expert has his own field and expertise, experts may make irrational comments. If the experts have the same weight, the result will be unreasonable. In addition, if Expert weights are set subjectively, especially in this multi-period situation, not only the workload is large, but also the deviation is large. Therefore, it is generally believed that the more deviated from the common cognition, the smaller the expert weight should be, and the closer to the common cognition, the greater the expert weight should be. For attribute weights, the attribute weights of each period are determined based on the 
idea of maximum deviation [26,27]. That is to say, the closer the evaluation value of the attribute is, the smaller the weight should be given. Otherwise, the greater weight should be given. Moreover, due to different evaluations by experts in different periods, attribute weights should also be different. For time weights, Guo et al. [28] proposed a combination of subjective and objective methods to determine the time weights. Subjective can comprehensively consider the expert's knowledge and experience, treat things with their own feelings, and make conclusions. Therefore, the combination of subjectivity and objectiveness can give more reasonable time weights.

In DIF-MAGDM, the proposed DIFPGWA operator not only aggregates the information of each stage, but also captures the fine nuances of the comprehensive evaluation value that the decision makers want to reflect. It is inaccurate to make a judgment based only on the evaluation from past to today. Based on this, this paper forecasts the comprehensive evaluation value of each scheme in the next period through the improved IFVs-GM(1,1)-PM. This method can make up for the lack of DIFPGWA operator. The proposed future adjustment coefficient can combine the two results to make the final result more accurate. Moreover, the multi-period weight determination method proposed in this paper also helps to obtain more accurate results. In the second chapter, this paper introduces the basic knowledge of intuitionistic fuzzy. In the third chapter, this paper constructs the operator and prediction model. In the fourth chapter, the paper gives the corresponding decision steps and predicting steps. In the fifth chapter, the decision method proposed in this paper is applied to the selection of the company's intern employees.

\section{Preliminaries}

\subsection{Concepts and Operational Rules}

Definition 1. IFS in space $X$ is defined as $A=\left\{\left[x, \mu_{A}(x), v_{A}(x)\right] \mid x \in X\right\}$. For any $x \in X, \mu_{A}(x)$ and $v_{A}(x)$ was expressed membership degree and non-membership degree of $A$. Moreover, $\mu_{A}(x)$ and $v_{A}(x)$ satisfy the following properties: $\mu_{A}: X \rightarrow[0,1], v_{A}: X \rightarrow[0,1]$ and $0 \leq \mu_{A}(x)+v_{A}(x) \leq 1, \forall x \in X$. For any $x \in X, \pi_{A}(x)=1-\mu_{A}(x)-v_{A}(x)$ was expressed as a hesitancy degree of $A$ [1].

For convenience of computation, IFVs was expressed as $\alpha=\left(\mu_{\alpha}, v_{\alpha}\right)$, where $\mu_{\alpha} \in[0,1], v_{\alpha} \in[0,1]$, $\mu_{\alpha}+v_{\alpha} \leq 1[10]$.

Definition 2. Let $\alpha=\left(\mu_{\alpha}, v_{\alpha}\right), \beta=\left(\mu_{\beta}, v_{\beta}\right)$ be IFVs, and the operational rules are as follows [19,29]:

$$
\begin{gathered}
\bar{\alpha}=\left(v_{\alpha}, \mu_{\alpha}\right), \\
\alpha \oplus \beta=\left(\mu_{\alpha}+\mu_{\beta}-\mu_{\alpha} \mu_{\beta}, v_{\alpha} v_{\beta}\right), \\
\lambda \alpha=\left(1-\left(1-\mu_{\alpha}\right)^{\lambda}, v_{\alpha}^{\lambda}\right), \lambda>0, \\
\alpha \otimes \beta=\left(\mu_{\alpha} \mu_{\beta}, v_{\alpha}+v_{\beta}-v_{\alpha} v_{\beta}\right), \\
\alpha^{\lambda}=\left(\mu_{\alpha}^{\lambda}, 1-\left(1-v_{\alpha}\right)^{\lambda}\right), \lambda>0, \\
d(\alpha, \beta)=\frac{1}{2}\left(\left|\mu_{\alpha}-\mu_{\beta}\right|+\left|v_{\alpha}-v_{\beta}\right|+\left|\left(1-\mu_{\alpha}-v_{\alpha}\right)-\left(1-\mu_{\beta}-v_{\beta}\right)\right|\right) . \\
=\frac{1}{2}\left(\left|\mu_{\alpha}-\mu_{\beta}\right|+\left|v_{\alpha}-v_{\beta}\right|+\left|\mu_{\alpha}-\mu_{\beta}+v_{\alpha}-v_{\beta}\right|\right)
\end{gathered}
$$

Definition 3. Support is a similarity indicator, the closer the two data values, the greater the support. Let $\alpha_{1}, \alpha_{2}, \cdots, \alpha_{n}$ be IFVs. Therefore, according to the character of support sets and the operation rules of IFVs, the support is calculated as follows [13]:

$$
\operatorname{Sup}\left(\alpha_{i}, \alpha_{j}\right)=1-\frac{d\left(\alpha_{i}, \alpha_{j}\right)}{\sum_{j=1, j \neq i}^{n} d\left(\alpha_{i}, \alpha_{j}\right)}
$$


$\operatorname{Sup}\left(\alpha_{i}, \alpha_{j}\right)$ express the support between $a_{i}$ and $\alpha_{j}$, and has the following three properties: $\operatorname{Sup}\left(\alpha_{i}, \alpha_{j}\right) \in[0,1]$; $\operatorname{Sup}\left(\alpha_{i}, \alpha_{j}\right)=\operatorname{Sup}\left(\alpha_{j}, \alpha_{i}\right)$. If $\left|\alpha_{i}-\alpha_{j}\right| \leq\left|\alpha_{s}-\alpha_{t}\right|$, then $\operatorname{Sup}\left(\alpha_{i}, \alpha_{j}\right) \geq \operatorname{Sup}\left(\alpha_{s}, \alpha_{t}\right)$.

2.2. The Comparing Method of IFVs

Definition 4. Let $\alpha=\left(\mu_{\alpha}, v_{\alpha}\right), \beta=\left(\mu_{\beta}, v_{\beta}\right)$ be IFVs, $s(\alpha)=\mu_{\alpha}-v_{\alpha}$ and $s(\beta)=\mu_{\beta}-v_{\beta}$ be the scores degrees of $\alpha$ and $\beta . h(\alpha)=\mu_{\alpha}+v_{\alpha}$ and $h(\beta)=\mu_{\beta}+v_{\beta}$ be the accuracy degrees of $\alpha$ and $\beta$; then,

- If $s(\alpha)<s(\beta)$, then $\alpha \prec \beta$,

- If $s(\alpha)>s(\beta)$, then $\alpha \succ \beta$,

- If $s(\alpha)=s(\beta)$, then if $h(\alpha)=h(\beta)$, then $\alpha=\beta$; if $h(\alpha)<h(\beta)$, then $\alpha \prec \beta$; if $h(\alpha)>h(\beta)$, then $\alpha \succ \beta[30,31]$.

\section{The Proposed IF-Operators and IFVs-GM(1,1)-PM}

\subsection{The IFPGWA Operator}

Definition 5. Let $a_{1}, a_{2}, \cdots, a_{n} \in R$; then, the geometric weighted average operators $W G A_{w}$ are defined as follows [32]:

$$
W G A_{w}\left(a_{1}, a_{2}, \cdots, a_{n}\right)=\prod_{i=1}^{n} a_{i}^{w_{i}}
$$

where $w=\left(w_{1}, w_{2}, \cdots, w_{n}\right)^{T}$ is the weight vector associated with the operator and $\forall w_{i} \geq 0, \sum_{i=1}^{n} w_{i}=1$.

Definition 6. Let $a_{1}, a_{2}, \cdots, a_{n} \in R$; then, the power geometric weighted average operators $P W G A_{w}$ is defined as follows [33]:

$$
P W G A_{w}\left(a_{1}, a_{2}, \cdots, a_{n}\right)=\prod_{i=1}^{n} a_{i}^{x_{i}}
$$

where $w=\left(w_{1}, w_{2}, \cdots, w_{n}\right)^{T}$ is the weight vector associated with the operator and $\forall w_{i} \geq 0, \sum_{i=1}^{n} w_{i}=1$, $T\left(a_{i}\right)=\sum_{j=1, j \neq i}^{n} w_{j} \operatorname{Sup}\left(a_{i}, a_{j}\right), x_{i}=\frac{w_{i}\left(1+T\left(a_{i}\right)\right)}{\sum_{i=1}^{n}\left(1+T\left(a_{i}\right)\right) w_{i}}$.

$\operatorname{Sup}\left(a_{i}, a_{j}\right)$ express the support between $a_{i}$ and $a_{j}$, and has the following three properties: $\operatorname{Sup}\left(a_{i}, a_{j}\right) \in$ $[0,1] ; \operatorname{Sup}\left(a_{i}, a_{j}\right)=\operatorname{Sup}\left(a_{j}, a_{i}\right) ;$ If $\operatorname{Sup}\left(a_{i}, a_{j}\right) \geq \operatorname{Sup}\left(a_{s}, a_{t}\right)$, then $\left|a_{i}-a_{j}\right| \leq\left|a_{s}-a_{t}\right|$.

Definition 7. Let $\alpha_{1}, \alpha_{2}, \cdots, \alpha_{n}$ be IFVs; then, the intuitionistic fuzzy power geometric weighted average (IFPGWA $A_{w}$ ) operator is defined as follows:

$$
\operatorname{IFPGW} A_{w}\left(\alpha_{1}, \alpha_{2}, \cdots, \alpha_{n}\right)=\prod_{i=1}^{n} \alpha_{i}^{\zeta_{i}}=\left(\prod_{i=1}^{n} \mu_{\alpha_{i}}^{\zeta_{i}}, 1-\prod_{i=1}^{n}\left(1-v_{\alpha_{i}}\right)^{\zeta_{i}}\right)
$$

where $T\left(\alpha_{i}\right)=\sum_{j=1, j \neq i}^{n} w_{j} \operatorname{Sup}\left(\alpha_{i}, \alpha_{j}\right), \operatorname{Sup}\left(\alpha_{i}, \alpha_{j}\right)=1-\frac{d\left(\alpha_{i}, \alpha_{j}\right)}{\sum_{j=1, j \neq i}^{n} d\left(\alpha_{i}, \alpha_{j}\right)}, \zeta_{i}=\frac{w_{i}\left(1+T\left(\alpha_{i}\right)\right)}{\sum_{i=1}^{n}\left(1+T\left(\alpha_{i}\right)\right) w_{i}}$.

It is easy to prove that the IFPGWA $A_{w}$ operator has the following properties:

Theorem 1. (Commutativity). Let $\left(\alpha_{1}, \alpha_{2}, \cdots, \alpha_{n}\right)$ be a vector of IFVs, and $\left(\alpha^{\prime}{ }_{1}, \alpha^{\prime}{ }_{2}, \cdots, \alpha^{\prime}{ }_{n}\right)$ be any permutation of $\left(\alpha_{1}, \alpha_{2}, \cdots, \alpha_{n}\right)$; then, $\operatorname{IFPGWA}_{w}\left(\alpha_{1}, \alpha_{2}, \cdots, \alpha_{n}\right)=\operatorname{IFPGWA_{w^{\prime }}}\left(\alpha^{\prime}{ }_{1}, \alpha^{\prime}{ }_{2}, \cdots, \alpha_{n}^{\prime}\right)$. 
Proof.

$$
\begin{gathered}
\operatorname{IFPGW} A_{w}\left(\alpha_{1}, \alpha_{2}, \cdots, \alpha_{n}\right)=\prod_{i=1}^{n} \alpha_{i}^{\zeta_{i}}=\left(\prod_{i=1}^{n} \mu_{\alpha_{i}}^{\zeta_{i}}, 1-\prod_{i=1}^{n}\left(1-v_{\alpha_{i}}\right)^{\zeta_{i}}\right) \\
=\left(\prod_{i=1}^{n} \mu_{\alpha^{\prime}{ }_{i}{ }^{\prime}}^{\zeta^{\prime}} 1-\prod_{i=1}^{n}\left(1-v_{\alpha^{\prime}}{ }_{i}\right)^{\zeta_{i}{ }^{\prime}}\right)=\prod_{i=1}^{n} \alpha_{i}^{\prime}{ }_{i}^{{ }^{\prime}}=\operatorname{IFPGW} A_{w^{\prime}}\left(\alpha^{\prime}{ }_{1}, \alpha^{\prime}{ }_{2}, \cdots, \alpha^{\prime}{ }_{n}\right)
\end{gathered}
$$

Theorem 2. (Idempotency). Let $\alpha_{j}(j=1,2, \cdots, n)$ be a collection of IFVs, if $\alpha_{j}=\alpha$, for all $j$; then, $\operatorname{IFPGW} A_{w}\left(\alpha_{1}, \alpha_{2}, \cdots, \alpha_{n}\right)=\alpha$.

Proof.

$$
\begin{aligned}
& \operatorname{IFPGWA} A_{w}\left(\alpha_{1}, \alpha_{2}, \cdots, \alpha_{n}\right)=\prod_{i=1}^{n} \alpha_{i}^{\zeta_{i}}=\left(\prod_{i=1}^{n} \mu_{\alpha_{i}}^{\zeta_{i}} 1-\prod_{i=1}^{n}\left(1-v_{\alpha_{i}}\right)^{\zeta_{i}}\right) \\
& =\left(\mu^{\sum_{i=1}^{n}\left(\frac{w^{\prime}{ }_{i}}{\sum_{i=1}^{n} w^{\prime}}\right)}, 1-(1-v)^{\sum_{i=1}^{n}\left(\frac{w^{\prime}}{\sum_{i=1}^{n} w^{\prime}}{ }_{i}\right.}\right)=(\mu, v)=\alpha
\end{aligned}
$$

Theorem 3. (Boundedness). Let $\alpha_{j}(j=1,2, \cdots, n)$ be a collection of IFVs, then $\alpha_{\text {min }} \leq$ $\operatorname{IFPGWA} A_{w}\left(\alpha_{1}, \alpha_{2}, \cdots, \alpha_{n}\right) \leq \alpha_{\max }$ where $\alpha_{\min }=\left(\min _{j}\left\{\mu_{\alpha_{j}}\right\}, \max _{j}\left\{v_{\alpha_{j}}\right\}\right)$ and $\alpha_{\max }=$ $\left(\max _{j}\left\{\mu_{\alpha_{j}}\right\}, \min _{j}\left\{v_{\alpha_{j}}\right\}\right)$.

Proof. According to the Theorem 2, the following inequalities can be proved:

$$
\begin{aligned}
& \operatorname{IFPGWA}_{w}\left(\alpha_{1}, \alpha_{2}, \cdots, \alpha_{n}\right) \geq \operatorname{IFPGW} A_{w}\left(\alpha_{\min }, \alpha_{\min }, \cdots, \alpha_{\min }\right)=\alpha_{\min } \\
& \operatorname{IFPGW} A_{w}\left(\alpha_{1}, \alpha_{2}, \cdots, \alpha_{n}\right) \leq \operatorname{IFPGWA} A_{w}\left(\alpha_{\max }, \alpha_{\max }, \cdots, \alpha_{\max }\right)=\alpha_{\max } .
\end{aligned}
$$

Then, $\alpha_{\min } \leq \operatorname{IFPGW} A_{w}\left(\alpha_{1}, \alpha_{2}, \cdots, \alpha_{n}\right) \leq \alpha_{\max }$ can be proved.

Example 1. Let $\alpha_{1}=(0.78,0.19), \alpha_{2}=(0.53,0.47), \alpha_{3}=(0.22,0.32)$ and $w_{1}=0.259, w_{2}=0.296$, $w_{3}=0.445$. The result obtained by IFPGWA $A_{w}$ operator is shown below:

$$
\begin{gathered}
T\left(\alpha_{1}\right)=0.346, T\left(\alpha_{2}\right)=0.330, T\left(\alpha_{3}\right)=0.279 ; \zeta_{1}=0.266, \zeta_{2}=0.300, \zeta_{3}=0.434 \\
\operatorname{IFPGWA}_{w}\left(\alpha_{1}, \alpha_{2}, \alpha_{3}\right)=(0.404,0.342) .
\end{gathered}
$$

\subsection{The DIFPGWA Operator}

Definition 8. Let $\alpha_{i}\left(t_{1}\right), \alpha_{i}\left(t_{2}\right), \cdots, \alpha_{i}\left(t_{g}\right)$ be the ith attribute value at different periods $t_{1}, t_{2}, \cdots, t_{g}$, where $\alpha_{i}\left(t_{l}\right)$ are expressed by IFVs. $\theta(t)=\left(\theta\left(t_{1}\right), \theta\left(t_{2}\right), \cdots, \theta\left(t_{g}\right)\right)^{T}$ be the weight vector of periods $t_{l}(l=1,2, \cdots, g)$ and $\theta\left(t_{l}\right) \geq 0, \sum_{l=1}^{g} \theta\left(t_{l}\right)=1, l=1,2, \cdots, g$; then, the dynamic intuitionistic fuzzy power geometric weighted average DIFPGW $A_{\lambda(t)}$ operator is defined as follows:

$$
\begin{gathered}
\operatorname{DIFPGW}_{\theta(t)}\left(\alpha_{i}\left(t_{1}\right), \alpha_{i}\left(t_{2}\right), \cdots, \alpha_{i}\left(t_{g}\right)\right)=\prod_{l=1}^{g}\left(\alpha_{i}\left(t_{l}\right)\right)^{\xi_{l}} \\
=\left(\prod_{l=1}^{g} \mu_{\alpha_{i}}^{\xi_{l}}\left(t_{l}\right), 1-\prod_{l=1}^{g}\left(1-v_{\alpha_{i}}\left(t_{l}\right)\right)^{\xi_{l}}\right)
\end{gathered}
$$


where $T\left(\alpha_{i}\left(t_{l}\right)\right)=\sum_{l=1, l \neq v}^{g} \theta\left(t_{l}\right) \operatorname{Sup}\left(\alpha_{i}\left(t_{l}\right), \alpha_{i}\left(t_{v}\right)\right), \operatorname{Sup}\left(\alpha_{i}\left(t_{l}\right), \alpha_{i}\left(t_{v}\right)\right)=1-\frac{d\left(\alpha_{i}\left(t_{l}\right), \alpha_{i}\left(t_{v}\right)\right)}{\sum_{l=1, l \neq v}^{g} d\left(\alpha_{i}\left(t_{l}\right), \alpha_{i}\left(t_{v}\right)\right)}$, $\xi_{l}=\frac{\theta\left(t_{l}\right)\left(1+T\left(\alpha_{i}\left(t_{l}\right)\right)\right)}{\sum_{l=1}^{g}\left(1+T\left(\alpha_{i}\left(t_{l}\right)\right)\right) \theta\left(t_{l}\right)}$.

It is easy to prove that the DIFPGWA $A_{\lambda(t)}$ operator has the three properties: Commutativity, Idempotency, and Boundedness. Here no longer proof.

Example 2. Let $\alpha_{i}\left(t_{1}\right)=(0.48,0.19), \alpha_{i}\left(t_{2}\right)=(0.77,0.10), \alpha_{i}\left(t_{3}\right)=(0.65,0.16)$ and $\theta\left(t_{1}\right)=0.2$, $\theta\left(t_{2}\right)=0.3, \theta\left(t_{3}\right)=0.5$. The result obtained by the DIFPGWA $A_{\theta(t)}$ operator is shown below:

$$
\begin{gathered}
T\left(\alpha_{i}\left(t_{1}\right)\right)=0.426, T\left(\alpha_{i}\left(t_{2}\right)\right)=0.413, T\left(\alpha_{i}\left(t_{3}\right)\right)=0.259 ; \xi_{1}=0.213, \xi_{2}=0.317, \xi_{3}=0.470 \\
\operatorname{DIFPGWA} A_{\theta(t)}\left(\alpha_{i}\left(t_{1}\right), \alpha_{i}\left(t_{2}\right), \alpha_{i}\left(t_{3}\right)\right)=(0.642,0.147) .
\end{gathered}
$$

\subsection{The Improved IFVs-GM(1,1)-PM}

Definition 9. Let $\alpha=\left(\alpha_{1}, \alpha_{2}, \cdots, \alpha_{n}\right)$ be IFVs time series; then, the IFV scores' degrees time series $S(\alpha)=$ $\left(S\left(\alpha_{1}\right), S\left(\alpha_{2}\right), \cdots, S\left(\alpha_{n}\right)\right)$ and IFVs' hesitancy degrees time series $\pi(\alpha)=\left(\pi\left(\alpha_{1}\right), \pi\left(\alpha_{2}\right), \cdots, \pi\left(\alpha_{n}\right)\right)$ can be calculated.

According to $S\left(\alpha_{k}\right)+\widehat{a} z^{(1)}(k)=b$, the time response function of $S(\alpha)=\left(S\left(\alpha_{1}\right), S\left(\alpha_{2}\right), \ldots, S\left(\alpha_{n}\right)\right)$ can be calculated as follows:

$$
S^{(1)}\left(\widehat{\alpha}_{k+1}\right)=\left(S\left(\alpha_{1}\right)-\frac{b}{a}\right) e^{-a k}+\frac{b}{a},
$$

where $z^{(1)}(k)=\frac{S\left(\alpha_{k}\right)+S\left(\alpha_{k-1}\right)}{2}$. Thus, the predictive value of IFVs scores degree can be calculated as follows:

$$
S\left(\widehat{\alpha}_{k+1}\right)=\left(1-e^{a}\right)\left(S\left(\alpha_{1}\right)-\frac{b}{a}\right) e^{-a k} .
$$

In addition, the predictive value of hesitancy degree can be calculated as follows:

$$
\pi\left(\widehat{\alpha}_{k+1}\right)=\max \left\{\pi\left(\alpha_{1}\right), \pi\left(\alpha_{2}\right), \cdots, \pi\left(\alpha_{n}\right)\right\} .
$$

According to the definition of IFVs, the IFVs-GM(1,1)-PM can be drawn as [24]

$$
\widehat{\alpha}_{k+1}=\left(\widehat{\mu}_{\alpha_{k+1}}, \widehat{v}_{\alpha_{k+1}}\right)=\left(\frac{1+S\left(\widehat{\alpha}_{k+1}\right)-\pi\left(\widehat{\alpha}_{k+1}\right)}{2}, \frac{1-S\left(\widehat{\alpha}_{k+1}\right)-\pi\left(\widehat{\alpha}_{k+1}\right)}{2}\right) .
$$

However, this IFVs-GM(1,1)-PM cannot ensure that the degree of membership and non-membership are both in the range of $[0,1]$. Therefore, the above IFVs-GM(1,1)-PM needs to be improved as follows:

$$
\begin{aligned}
\widehat{\alpha}_{k+1}=\left(\widehat{\mu}_{\alpha_{k+1}}, \widehat{v}_{\alpha_{k+1}}\right)= & \left(\min \left(\left(\max \left(\frac{1+S\left(\widehat{\alpha}_{k+1}\right)-\pi\left(\widehat{\alpha}_{k+1}\right)}{2}, 0\right)\right), 1\right), \rightarrow\right. \\
& \left.\rightarrow \min \left(\left(\max \left(\frac{1-S\left(\widehat{\alpha}_{k+1}\right)-\pi\left(\widehat{\alpha}_{k+1}\right)}{2}, 0\right)\right), 1\right)\right)
\end{aligned}
$$

The best using condition of IFVs-GM(1,1)-PM is: $a \geq-0.5$ [24].

Example 3. Let $\alpha_{1}=(0.58,0.17), \alpha_{2}=(0.63,0.19), \alpha_{3}=(0.72,0.20)$ be IFVs' time series. IFV scores' degrees time series $S(\alpha)=(0.41,0.44,0.52)$ and IFVs hesitancy degrees time series $\pi(\alpha)=(0.25,0.18,0.08)$. The result obtained by IFVs-GM(1,1)-PM is: $\widehat{\alpha}_{4}=(0.681,0.069)$. 


\subsection{Future Adjustment Coefficient}

Definition 10. Let $\psi$ be future adjustment coefficient in the range of $[0,1]$. Assuming that $\alpha=\left(\mu_{\alpha}, v_{\alpha}\right)$ is the aggregated decision value in period of $[0, t], \beta=\left(\mu_{\beta}, v_{\beta}\right)$ is the predicted decision value in the period $t+1$. The formula of the comprehensive evaluation value is as follows:

$$
\begin{aligned}
& \phi=(1-\psi) \alpha \oplus \psi \beta=\left(1-\left(1-\mu_{\alpha}\right)^{(1-\psi)}, v_{\alpha}^{(1-\psi)}\right) \oplus\left(1-\left(1-\mu_{\beta}\right)^{\psi}, v_{\beta}^{\psi}\right) \\
& \left(\left(1-\left(1-\mu_{\alpha}\right)^{(1-\psi)}\right)+\left(1-\left(1-\mu_{\beta}\right)^{\psi}\right)-\left(1-\left(1-\mu_{\alpha}\right)^{(1-\psi)}\right)\left(1-\left(1-\mu_{\beta}\right)^{\psi}\right), v_{\alpha}^{(1-\psi)} v_{\beta}^{\psi}\right)
\end{aligned}
$$

Example 4. Suppose there are two sets of time series, as shown in Table 1.

Table 1. Time series A and B.

\begin{tabular}{cccc}
\hline Time Series A & Scores Degree of A & Time Series B & Scores Degree of B \\
\hline$(0.4,0.1)$ & 0.3 & $(0.60,0.10)$ & 0.50 \\
$(0.5,0.1)$ & 0.4 & $(0.55,0.10)$ & 0.45 \\
$(0.6,0.1)$ & 0.5 & $(0.50,0.10)$ & 0.40 \\
\hline
\end{tabular}

The time weight vector is $(0.2,0.3,0.5)$. By observing the score degree of the Time series $A$ and $B$, it is obvious that $A$ is in the rising period and $B$ is in the recession period. The results shown in Table 2, which is calculated by DIFPGWA $\lambda_{(t)}$ operator IFVs-GM(1,1)-PM and Future Adjustment Coefficient. Let $\psi=0.5$.

Table 2. Results of time series A and B.

\begin{tabular}{cccc}
\hline Time Series & Type & Result & Scores Degree \\
\hline \multirow{2}{*}{ A } & Result of aggregation & $(0.52,0.10)$ & 0.42 \\
& Result of prediction & $(0.50,0.00)$ & 0.50 \\
& comprehensive evaluation & $(0.51,0.00)$ & 0.51 \\
\hline \multirow{2}{*}{ B } & Result of aggregation & $(0.53,0.10)$ & 0.43 \\
& Result of prediction & $(0.48,0.12)$ & 0.36 \\
& comprehensive evaluation & $(0.506,0.11)$ & 0.396 \\
\hline
\end{tabular}

The result of using the DIFPGWA $A_{\lambda(t)}$ operator is $A \prec B$. The result obtained using the IFVs-GM(1,1)-PM is $A \succ B$. The comprehensive evaluation is $A \succ B$. Obviously, the results obtained using the IFVs-GM(1,1)-PM are more accurate. However, the advantage of the DIFPGW $A_{\lambda(t)}$ operator could not be negated. There are also errors in the IFVs-GM(1,1)-PM. Obviously, through the adjustment coefficient in the future, the results of the two can be integrated to get more accurate comprehensive evaluation value.

\section{Decision-Making Steps}

Description 1. In a MAGDM process, $E=\left\{e_{k} \mid k=1,2, \cdots, p\right\}$ is a set of experts, $A=\left\{A_{i} \mid i=1,2, \cdots, m\right\}$ is an alternative set, and $C=\left\{C_{j} \mid j=1,2, \cdots, n\right\}$ is a set of attributes. Expert's evaluation values are expressed by IFVs. The weight vector of time under different period $t_{l}$ is $\theta(t)$, and $\theta(t)=\left(\theta\left(t_{1}\right), \theta\left(t_{2}\right), \cdots, \theta\left(t_{g}\right)\right)^{T}$, $\theta\left(t_{l}\right) \geq 0, \sum_{l=1}^{g} \theta\left(t_{l}\right)=1, l=1,2, \cdots, g$. The attribute weight vector in period $t_{l}$ is $w\left(t_{l}\right)=$ $\left(w_{1}\left(t_{l}\right), w_{2}\left(t_{l}\right), \cdots, w_{n}\left(t_{l}\right)\right)^{T}, l=1,2, \cdots, g$, where $w_{j}\left(t_{l}\right) \geq 0, \sum_{j=1}^{n} w_{k}=1$. The expert weight vector in period $t_{l}$ is $\lambda\left(t_{l}\right)=\left(\lambda_{1}\left(t_{l}\right), \lambda_{2}\left(t_{l}\right), \cdots, \lambda_{p}\left(t_{l}\right)\right)^{T}, l=1,2, \cdots, g$, where $\lambda_{k}\left(t_{l}\right) \geq 0, \sum_{k=1}^{p} \lambda_{k}=1$. The evaluation matrix made by expert $e_{k}$ at time $t_{l}$ is $A_{i j k}\left(t_{l}\right)=\left(\alpha_{i j k}\left(t_{l}\right)\right)_{m \times n}$. 
- Step 1. Determine expert weights and group decision matrix

Because each expert has his own field and expertise, experts may make irrational comments. If the experts have the same weight, the result will be unreasonable. On the other hand, if expert weights are set subjectively, especially in this multi-period situation, not only the workload is large, but also the deviation is large. Therefore, the following group mean evaluation matrix $A^{\prime}{ }_{i j}\left(t_{l}\right)$ is constructed. Assessments close to the average will have a greater weight, while assessments away from the average will have smaller weight [25]:

$$
A_{i j}^{\prime}\left(t_{l}\right)=\left(\alpha_{i j}^{\prime}\left(t_{l}\right)\right)_{m \times n}=\left(\mu_{i j}^{\prime}\left(t_{l}\right), v_{i j}^{\prime}\left(t_{l}\right)\right)=\left(\frac{1}{p} \sum_{k=1}^{p} \mu_{i j k}\left(t_{l}\right), \frac{1}{p} \sum_{k=1}^{p} v_{i j k}\left(t_{l}\right)\right) .
$$

The degree of similarity between $\alpha_{i j k}\left(t_{l}\right), k=1,2, \ldots, p$ and $\alpha_{i j}^{\prime}\left(t_{l}\right)$ is defined as $S\left(\alpha_{i j k}\left(t_{l}\right), \alpha_{i j}^{\prime}\left(t_{l}\right)\right)$

$$
S\left(\alpha_{i j k}\left(t_{l}\right), \alpha^{\prime}{ }_{i j}\left(t_{l}\right)\right)=1-\frac{d\left(\alpha_{i j k}\left(t_{l}\right), \alpha^{\prime}{ }_{i j}\left(t_{l}\right)\right)}{\sum_{k=1}^{p} d\left(\alpha_{i j k}\left(t_{l}\right), \alpha^{\prime}{ }_{i j}\left(t_{l}\right)\right)}, k=1,2, \cdots, p, i=1,2, \cdots, m, j=1,2, \cdots, n,
$$

where $\sum_{k=1}^{p} d\left(\alpha_{i j k}\left(t_{l}\right), \alpha^{\prime}{ }_{i j}\left(t_{l}\right)\right) \neq 0$.

If $\sum_{k=1}^{p} d\left(\alpha_{i j k}\left(t_{l}\right), \alpha_{i j}^{\prime}\left(t_{l}\right)\right)=0$, then $S\left(\alpha_{i j k}\left(t_{l}\right), \alpha_{i j}^{\prime}\left(t_{l}\right)\right)=1-\frac{1}{p}$.

Then, the expert weight for $\lambda_{i j k}\left(t_{l}\right)$ is defined as:

$$
\lambda_{i j k}\left(t_{l}\right)=\frac{S\left(\alpha_{i j k}\left(t_{l}\right), \alpha^{\prime}{ }_{i j}\left(t_{l}\right)\right)}{\sum_{k=1}^{p} S\left(\alpha_{i j k}\left(t_{l}\right), \alpha^{\prime}{ }_{i j}\left(t_{l}\right)\right)}, k=1,2, \cdots, p, i=1,2, \cdots, m, j=1,2, \cdots, n .
$$

In period $t_{l}$, the group decision matrix $B_{i j}\left(t_{l}\right)=\left(\beta_{i j}\left(t_{l}\right)\right)_{m \times n}$ is obtained by aggregating the information of each expert with IFPGW $A_{\lambda_{i j k}\left(t_{l}\right)}$ operator, where

$$
\begin{aligned}
& \beta_{i j}\left(t_{l}\right)=I F P G W A_{\lambda_{i j k}\left(t_{l}\right)}\left(\alpha_{i j 1}\left(t_{l}\right), \alpha_{i j 2}\left(t_{l}\right), \cdots, \alpha_{i j p}\left(t_{l}\right)\right)=\prod_{k=1}^{p} \alpha_{i j k}^{\zeta_{k}}\left(t_{l}\right) \\
& =\left(\prod_{k=1}^{p} \mu_{\alpha_{i j k}}^{\zeta_{k}}\left(t_{l}\right), 1-\prod_{k=1}^{p}\left(1-v_{\alpha_{i j k}}\left(t_{l}\right)\right)^{\zeta_{k}}\right)
\end{aligned}
$$

where $T\left(\alpha_{i j k}\left(t_{l}\right)\right)=\sum_{k=1, k \neq x}^{p} \lambda_{i j k}\left(t_{l}\right) \operatorname{Sup}\left(\alpha_{i j k}\left(t_{l}\right), \alpha_{i j x}\left(t_{l}\right)\right), \quad \operatorname{Sup}\left(\alpha_{i j k}\left(t_{l}\right), \alpha_{i j x}\left(t_{l}\right)\right)=1-$ $\frac{d\left(\alpha_{i j k}\left(t_{l}\right), \alpha_{i j x}\left(t_{l}\right)\right)}{\sum_{k=1, k \neq x}^{p} d\left(\alpha_{i j k}\left(t_{l}\right), \alpha_{i j x}\left(t_{l}\right)\right)}, \zeta_{k}=\frac{\lambda_{i j k}\left(t_{l}\right)\left(1+T\left(\alpha_{i j k}\left(t_{l}\right)\right)\right)}{\sum_{k=1}^{p}\left(1+T\left(\alpha_{i j k}\left(t_{l}\right)\right)\right) \lambda_{i j k}\left(t_{l}\right)}$.

- Step 2. Determine attribute weights

In period $t_{l}$, for attribute $C_{j}$, specifies that $D_{i j}\left(w_{j}\left(t_{l}\right)\right)=\sum_{l=1}^{m} d\left(\beta_{i j}\left(t_{l}\right), \beta_{s j}\left(t_{l}\right)\right) w_{j}\left(t_{l}\right)$ represents the total deviation.

Then, for all attributes $D\left(w_{j}\left(t_{l}\right)\right)=\sum_{j=1}^{n} \sum_{i=1}^{m} D_{i j}\left(w_{j}\left(t_{l}\right)\right)=\sum_{j=1}^{n} \sum_{i=1}^{m} \sum_{l=1}^{m} d\left(\beta_{i j}\left(t_{l}\right), \beta_{s j}\left(t_{l}\right)\right) w_{j}\left(t_{l}\right)$ represents the total deviation. Then, the following model is constructed: 


$$
\begin{aligned}
& \max D\left(w_{j}\left(t_{l}\right)\right)=\sum_{j=1}^{n} \sum_{i=1}^{m} D_{i j}\left(w_{j}\left(t_{l}\right)\right)=\sum_{j=1}^{n} \sum_{i=1}^{m} \sum_{l=1}^{m} d\left(\beta_{i j}\left(t_{l}\right), \beta_{s j}\left(t_{l}\right)\right) w_{j}\left(t_{l}\right) \\
& \text { s.t. }\left\{\begin{array}{c}
\sum_{j=1}^{n}\left(w_{j}\left(t_{l}\right)\right)^{2}=1 \\
w_{j}\left(t_{l}\right) \geq 0, j=1,2, \cdots, n
\end{array}\right.
\end{aligned}
$$

According to Label (22), the results are shown below:

$$
w_{j}\left(t_{l}\right)=\frac{\sum_{i=1}^{m} \sum_{l=1}^{m} d\left(\beta_{i j}\left(t_{l}\right), \beta_{s j}\left(t_{l}\right)\right)}{\sqrt{\sum_{j=1}^{n}\left(\sum_{i=1}^{m} \sum_{l=1}^{m} d\left(\beta_{i j}\left(t_{l}\right), \beta_{s j}\left(t_{l}\right)\right)\right)^{2}}} .
$$

Then, the results of standardization are as follows:

$$
w_{j}\left(t_{l}\right)=\frac{\sum_{i=1}^{m} \sum_{l=1}^{m} d\left(\beta_{i j}\left(t_{l}\right), \beta_{s j}\left(t_{l}\right)\right)}{\sum_{j=1}^{n} \sum_{i=1}^{m} \sum_{l=1}^{m} d\left(\beta_{i j}\left(t_{l}\right), \beta_{s j}\left(t_{l}\right)\right)}
$$

Therefore, the attribute weight vector in period $t_{l}$ is $w\left(t_{l}\right)=\left(w_{1}\left(t_{l}\right), w_{2}\left(t_{l}\right), \cdots, w_{n}\left(t_{l}\right)\right)^{T}$, $l=1,2, \cdots, g$, where $w_{j}\left(t_{l}\right) \geq 0, \sum_{j=1}^{n} w_{k}=1$.

- Step 3. Determine all alternative's comprehensive evaluation value by operator in each period

All the alternative's comprehensive evaluation value by operator $X\left(t_{l}\right)=\left(\chi_{1}\left(t_{l}\right), \chi_{2}\left(t_{l}\right), \cdots, \chi_{m}\left(t_{l}\right)\right)^{T}$ is obtained by aggregating the information of each attribute with IFPGW $A_{w_{j}\left(t_{l}\right)}$ operator in period $t_{l}$, $l=1,2, \cdots, g$, where

$$
\begin{gathered}
\chi_{i}\left(t_{l}\right)=\operatorname{IFPGW} A_{w_{j}\left(t_{l}\right)}\left(\beta_{i 1}\left(t_{l}\right), \beta_{i 2}\left(t_{l}\right), \cdots, \beta_{i n}\left(t_{l}\right)\right)=\prod_{j=1}^{n} \beta_{i j}^{\zeta_{j}}\left(t_{l}\right) \\
=\left(\prod_{j=1}^{n} \mu_{\beta_{i j}}^{\zeta_{j}}\left(t_{l}\right), 1-\prod_{j=1}^{n}\left(1-v_{\beta_{i j}}\left(t_{l}\right)\right)^{\zeta_{j}}\right)
\end{gathered}
$$

where $T\left(\beta_{i j}\left(t_{l}\right)\right)=\sum_{j=1, j \neq s}^{n} w_{j}\left(t_{l}\right) \operatorname{Sup}\left(\beta_{i j}\left(t_{l}\right), \beta_{s j}\left(t_{l}\right)\right), \operatorname{Sup}\left(\beta_{i j}\left(t_{l}\right), \beta_{s j}\left(t_{l}\right)\right)=1-\frac{d\left(\beta_{i j}\left(t_{l}\right), \beta_{s j}\left(t_{l}\right)\right)}{\sum_{j=1, j \neq s}^{n} d\left(\beta_{i j}\left(t_{l}\right), \beta_{s j}\left(t_{l}\right)\right)}$, $\zeta_{j}=\frac{w_{j}\left(t_{l}\right)\left(1+T\left(\beta_{i j}\left(t_{l}\right)\right)\right)}{\sum_{j=1}^{n}\left(1+T\left(\beta_{i j}\left(t_{l}\right)\right)\right) w_{j}\left(t_{l}\right)}$.

- $\quad$ Step 4. Determine time weights

In order to prevent the error caused by the weight setting of the supervisor and objective, the two methods should be used together. The time weight is obtained as follows [28]:

$$
\begin{gathered}
\max \left[-\sum_{l=1}^{g} \theta\left(t_{l}\right) \ln \left(\theta\left(t_{l}\right)\right)\right] \\
\delta=-\sum_{l=1}^{g}\left(\frac{g-l}{g-1} \theta\left(t_{l}\right)\right) \\
\text { s.t. }\left\{\begin{array}{c}
g \\
\sum_{l=1}^{g} \theta\left(t_{l}\right)=1, \theta\left(t_{l}\right) \in[0,1], g=1,2, \cdots, p
\end{array}\right.
\end{gathered}
$$

$\theta(t)=\left(\theta\left(t_{1}\right), \theta\left(t_{2}\right), \cdots, \theta\left(t_{g}\right)\right)^{T}$ are the weight of each period and $\theta\left(t_{l}\right) \geq 0, \sum_{l=1}^{g} \theta\left(t_{l}\right)=1$. 
$\delta$ represents subjective emphasis on recent data. $\delta \in[0,1]$, if $\delta$ is closer to 1 , there is more emphasis on recent data. If $\delta$ is closer to 0 , there is more emphasis placed on forward data.

- Step 5. Determine all alternative's comprehensive evaluation value by operator

All alternative's comprehensive evaluation value by operator $Y=\left(Y_{1}, Y_{2}, \cdots, Y_{m}\right)^{T}$ is obtained by aggregating the information of each attribute with $D I F P G W A_{\theta(t)}$ operator, where

$$
\begin{aligned}
& Y_{i}=\operatorname{DIFPGWA}_{\theta(t)}\left(\chi_{i}\left(t_{1}\right), \chi_{i}\left(t_{2}\right), \cdots, \chi_{i}\left(t_{g}\right)\right)=\prod_{l=1}^{g}\left(\chi_{i}\left(t_{l}\right)\right)^{\xi_{l}}, \\
& =\left(\prod_{l=1}^{g} \mu_{\chi_{i}}^{\xi_{l}}\left(t_{l}\right), 1-\prod_{l=1}^{g}\left(1-v_{\chi_{i}}\left(t_{l}\right)\right)^{\xi_{l}}\right)
\end{aligned}
$$

where $T\left(\chi_{i}\left(t_{l}\right)\right)=\sum_{l=1, l \neq v}^{g} \theta\left(t_{l}\right) \operatorname{Sup}\left(\chi_{i}\left(t_{l}\right), \chi_{i}\left(t_{v}\right)\right), \operatorname{Sup}\left(\chi_{i}\left(t_{l}\right), \chi_{i}\left(t_{v}\right)\right)=1-\frac{d\left(\chi_{i}\left(t_{l}\right), \chi_{i}\left(t_{v}\right)\right)}{\sum_{l=1, l \neq v}^{g} d\left(\chi_{i}\left(t_{l}\right), \chi_{i}\left(t_{v}\right)\right)}$, $\xi_{l}=\frac{\theta\left(t_{l}\right)\left(1+T\left(\chi_{i}\left(t_{l}\right)\right)\right)}{\sum_{l=1}^{g}\left(1+T\left(\chi_{i}\left(t_{l}\right)\right)\right) \theta\left(t_{l}\right)}$.

- Step 6. Predicting the $g+1$ period comprehensive evaluation value

In Step 3, all alternative's comprehensive evaluation value is calculated by operator $X\left(t_{l}\right)=$ $\left(\chi_{1}\left(t_{l}\right), \chi_{2}\left(t_{l}\right), \cdots, \chi_{m}\left(t_{l}\right)\right)^{T}, l=1,2, \cdots, g$, which is $X_{i}=\left(\chi_{i}\left(t_{1}\right), \chi_{i}\left(t_{2}\right), \cdots, \chi_{i}\left(t_{g}\right)\right), i=1,2, \cdots, m$.

Then, the IFVs scores degrees time series $S\left(X_{i}\right)=\left(S\left(\chi_{i}\left(t_{1}\right)\right), S\left(\chi_{i}\left(t_{2}\right)\right), \cdots, S\left(\chi_{i}\left(t_{g}\right)\right)\right)$, $i=1,2, \cdots, m$ and the IFV hesitancy degrees time series $\pi\left(X_{i}\right)=\left(\pi\left(\chi_{i}\left(t_{1}\right)\right), \pi\left(\chi_{i}\left(t_{2}\right)\right), \cdots, \pi\left(\chi_{i}\left(t_{g}\right)\right)\right)$, $i=1,2, \cdots, m$ could be calculated.

According to $S\left(\chi_{i}\left(t_{k}\right)\right)+\widehat{a} z^{(1)}(k)=b$, the time response function of $S\left(X_{i}\right)=$ $\left(S\left(\chi_{i}\left(t_{1}\right)\right), S\left(\chi_{i}\left(t_{2}\right)\right), \cdots, S\left(\chi_{i}\left(t_{g}\right)\right)\right)$ is $S^{(1)}\left(\widehat{\chi}_{i}\left(t_{g+1}\right)\right)=\left(S\left(\chi_{i}\left(t_{1}\right)\right)-\frac{b}{a}\right) e^{-a g}+\frac{b}{a}$, where $z^{(1)}(k)=$ $\frac{S\left(\chi_{i}\left(t_{k}\right)\right)+S\left(\chi_{i}\left(t_{k-1}\right)\right)}{2}$. Thus, the predictive value of IFVs scores degree can be calculated as follows:

$$
S\left(\widehat{\chi}_{i}\left(t_{g+1}\right)\right)=\left(1-e^{a}\right)\left(S\left(\chi_{i}\left(t_{1}\right)\right)-\frac{b}{a}\right) e^{-a g}
$$

In addition, the predictive value of hesitancy degree is $\pi\left(\widehat{\chi}_{i}\left(t_{g+1}\right)\right)=$ $\max \left\{\pi\left(\chi_{i}\left(t_{1}\right)\right), \pi\left(\chi_{i}\left(t_{2}\right)\right), \cdots, \pi\left(\chi_{i}\left(t_{g}\right)\right)\right\}$.

According to the definition of IFVs, the IFVs-GM(1,1)-PM can be calculated as follows:

$$
\begin{aligned}
& \widehat{\chi}_{i}\left(t_{g+1}\right)=\left(\widehat{\mu}_{\chi_{i}\left(t_{g+1}\right)}, \widehat{v}_{\chi_{i}\left(t_{g+1}\right)}\right) \\
& =\left(\min \left(\max \left(\frac{1+S\left(\widehat{\chi}_{i}\left(t_{g+1}\right)\right)-\pi\left(\widehat{\chi}_{i}\left(t_{g+1}\right)\right)}{2}, 0\right), 1\right), \rightarrow .\right. \\
& \left.\rightarrow \min \left(\max \left(\frac{1-S\left(\hat{\chi}_{i}\left(t_{g+1}\right)\right)-\pi\left(\widehat{\chi}_{i}\left(t_{g+1}\right)\right)}{2}, 0\right), 1\right)\right)
\end{aligned}
$$

Then, all alternative's predictive comprehensive evaluation value are obtained as follows:

$$
\widehat{Y}=\left(\widehat{\chi}_{1}\left(t_{g+1}\right), \widehat{\chi}_{2}\left(t_{g+1}\right), \cdots, \widehat{\chi}_{m}\left(t_{g+1}\right)\right)^{T}=\left(\widehat{Y}_{1}, \widehat{Y}_{2}, \cdots, \widehat{Y}_{m}\right)^{T}
$$

\section{- $\quad$ Step 7. Comprehensive evaluation}

Let $\psi$ be future adjustment coefficient in the range of $[0,1]$. The formula for calculating the comprehensive evaluation values of all alternatives is as follows: 


$$
\bar{Y}=\left((1-\psi) Y_{1} \oplus \psi \widehat{Y}_{1},(1-\psi) Y_{2} \oplus \psi \widehat{Y}_{2}, \cdots,(1-\psi) Y_{m} \oplus \psi \widehat{Y}_{m}\right)^{T}=\left(\bar{Y}_{1}, \bar{Y}_{2}, \cdots, \bar{Y}_{m}\right)^{T}
$$

All alternatives are sorted according to the ranking method of IFVs. If $\max \left(\bar{Y}_{1}, \bar{Y}_{2}, \cdots, \bar{Y}_{m}\right)=\bar{Y}_{i}$, the $i$ th alternative is best in period $g+1$. If $\max \left(\bar{Y}_{1}, \cdots, \bar{Y}_{i-1}, \bar{Y}_{i+1}, \cdots, \bar{Y}_{m}\right)=\bar{Y}_{j}$, then the $j$ th alternative is suboptimal in now.

\section{Example}

A company plans to recruit two employees. A total of five interviewees $\left(A_{1}, A_{2}, A_{3}, A_{4}, A_{5}\right)$ received internship opportunities. The internship lasted for three months $\left(t_{1}, t_{2}, t_{3}\right)$. At the end of each month, three experts $\left(e_{1}, e_{2}, e_{3}\right)$ evaluated their performance during the month. These candidates $\left(A_{1}, A_{2}, A_{3}, A_{4}, A_{5}\right)$ are evaluated in terms of practical ability $C_{1}$, learning ability $C_{2}$ and innovation ability $C_{3}$. Each expert uses the IFVs to evaluate five candidates in each period, as shown in Tables 3-5.

Table 3. The three experts' evaluation value in the first month $t_{1}$.

\begin{tabular}{ccccccc}
\hline \multicolumn{2}{c}{ Period $t_{1}$} & $A_{1}$ & $A_{2}$ & $A_{3}$ & $A_{4}$ & $A_{5}$ \\
\hline \multirow{6}{*}{ Experts $e_{1}$} & $C_{1}$ & $(0.56,0.33)$ & $(0.56,0.16)$ & $(0.78,0.13)$ & $(0.73,0.27)$ & $(0.88,0.12)$ \\
& $C_{2}$ & $(0.57,0.32)$ & $(0.58,0.16)$ & $(0.74,0.17)$ & $(0.48,0.52)$ & $(0.82,0.18)$ \\
& $C_{3}$ & $(0.57,0.31)$ & $(0.61,0.17)$ & $(0.83,0.07)$ & $(0.02,0.40)$ & $(0.36,0.24)$ \\
\hline \multirow{4}{*}{ Experts $e_{2}$} & $C_{1}$ & $(0.63,0.34)$ & $(0.65,0.16)$ & $(0.82,0.07)$ & $(0.72,0.14)$ & $(0.77,0.16)$ \\
& $C_{2}$ & $(0.63,0.35)$ & $(0.64,0.16)$ & $(0.81,0.08)$ & $(0.70,0.15)$ & $(0.58,0.42)$ \\
& $C_{3}$ & $(0.57,0.31)$ & $(0.63,0.17)$ & $(0.78,0.13)$ & $(0.78,0.22)$ & $(0.16,0.47)$ \\
\hline \multirow{6}{*}{ Experts $e_{3}$} & $C_{1}$ & $(0.66,0.33)$ & $(0.52,0.17)$ & $(0.80,0.09)$ & $(0.58,0.42)$ & $(0.70,0.30)$ \\
& $C_{2}$ & $(0.63,0.32)$ & $(0.53,0.16)$ & $(0.79,0.09)$ & $(0.76,0.11)$ & $(0.28,0.72)$ \\
& $C_{3}$ & $(0.63,0.34)$ & $(0.48,0.19)$ & $(0.77,0.10)$ & $(0.65,0.16)$ & $(0.19,0.25)$ \\
\hline
\end{tabular}

Table 4. The three experts' evaluation value in the second month $t_{2}$.

\begin{tabular}{ccccccc}
\hline \multicolumn{2}{c}{ Period $t_{2}$} & $A_{1}$ & $A_{2}$ & $A_{3}$ & $A_{4}$ & $A_{5}$ \\
\hline \multirow{6}{*}{ Experts $e_{1}$} & $C_{1}$ & $(0.62,0.33)$ & $(0.51,0.17)$ & $(0.75,0.12)$ & $(0.50,0.50)$ & $(0.57,0.11)$ \\
& $C_{2}$ & $(0.60,0.35)$ & $(0.51,0.17)$ & $(0.72,0.14)$ & $(0.85,0.15)$ & $(0.85,0.15)$ \\
& $C_{3}$ & $(0.66,0.32)$ & $(0.62,0.17)$ & $(0.70,0.14)$ & $(0.74,0.13)$ & $(0.41,0.06)$ \\
\hline \multirow{4}{*}{ Experts $e_{2}$} & $C_{1}$ & $(0.61,0.32)$ & $(0.61,0.32)$ & $(0.68,0.15)$ & $(0.40,0.39)$ & $(0.40,0.60)$ \\
& $C_{2}$ & $(0.55,0.18)$ & $(0.65,0.16)$ & $(0.67,0.16)$ & $(0.74,0.26)$ & $(0.24,0.31)$ \\
& $C_{3}$ & $(0.53,0.16)$ & $(0.61,0.32)$ & $(0.64,0.16)$ & $(0.87,0.13)$ & $(0.84,0.16)$ \\
\hline \multirow{4}{*}{ Experts $e_{3}$} & $C_{1}$ & $(0.77,0.10)$ & $(0.71,0.14)$ & $(0.68,0.15)$ & $(0.44,0.20)$ & $(0.72,0.28)$ \\
& $C_{2}$ & $(0.71,0.14)$ & $(0.74,0.13)$ & $(0.72,0.13)$ & $(0.51,0.18)$ & $(0.75,0.25)$ \\
& $C_{3}$ & $(0.70,0.16)$ & $(0.77,0.10)$ & $(0.63,0.17)$ & $(0.70,0.30)$ & $(0.43,0.39)$ \\
\hline
\end{tabular}

Table 5. The three experts' evaluation value in the third month $t_{3}$.

\begin{tabular}{ccccccc}
\hline \multicolumn{2}{c}{ Period $t_{3}$} & $A_{1}$ & $A_{2}$ & $A_{3}$ & $A_{4}$ & $A_{5}$ \\
\hline \multirow{2}{*}{ Experts $e_{1}$} & $C_{1}$ & $(0.62,0.32)$ & $(0.72,0.14)$ & $(0.56,0.16)$ & $(0.39,0.61)$ & $(0.91,0.09)$ \\
& $C_{2}$ & $(0.72,0.14)$ & $(0.83,0.07)$ & $(0.53,0.17)$ & $(0.13,0.33)$ & $(0.15,0.53)$ \\
& $C_{3}$ & $(0.68,0.25)$ & $(0.62,0.32)$ & $(0.54,0.16)$ & $(0.63,0.21)$ & $(0.07,0.16)$ \\
\hline \multirow{4}{*}{ Experts $e_{2}$} & $C_{1}$ & $(0.62,0.32)$ & $(0.67,0.25)$ & $(0.55,0.16)$ & $(0.47,0.11)$ & $(0.87,0.02)$ \\
& $C_{2}$ & $(0.67,0.25)$ & $(0.61,0.32)$ & $(0.49,0.18)$ & $(0.41,0.49)$ & $(0.35,0.30)$ \\
& $C_{3}$ & $(0.61,0.32)$ & $(0.74,0.17)$ & $(0.40,0.22)$ & $(0.21,0.44)$ & $(0.92,0.08)$ \\
\hline \multirow{4}{*}{ Experts $e_{3}$} & $C_{1}$ & $(0.82,0.13)$ & $(0.61,0.32)$ & $(0.38,0.23)$ & $(0.42,0.50)$ & $(0.51,0.21)$ \\
& $C_{2}$ & $(0.83,0.13)$ & $(0.81,0.13)$ & $(0.36,0.24)$ & $(0.14,0.59)$ & $(0.81,0.19)$ \\
& $C_{3}$ & $(0.62,0.32)$ & $(0.82,0.13)$ & $(0.37,0.23)$ & $(0.23,0.65)$ & $(0.26,0.74)$ \\
\hline
\end{tabular}


- Step 1. Based on the above evaluation information given by the experts in each period, the group mean evaluation matrix could be constructed. Then, using Equations (18)-(20), to get the expert weights. The group decision matrices for each period are calculated by Equation (21), which is shown in Table 6.

Table 6. Group decision matrix in each month.

\begin{tabular}{|c|c|c|c|c|c|c|}
\hline & & $A_{1}$ & $A_{2}$ & $A_{3}$ & $A_{4}$ & $A_{5}$ \\
\hline \multirow{3}{*}{ Period $t_{1}$} & $C_{1}$ & $(0.62,0.33)$ & $(0.57,0.16)$ & $(0.80,0.09)$ & $(0.68,0.28)$ & $(0.78,0.19)$ \\
\hline & $C_{2}$ & $(0.61,0.33)$ & $(0.58,0.16)$ & $(0.78,0.11)$ & $(0.65,0.25)$ & $(0.53,0.47)$ \\
\hline & $C_{3}$ & $(0.58,0.31)$ & $(0.58,0.17)$ & $(0.79,0.10)$ & $(0.29,0.25)$ & $(0.22,0.32)$ \\
\hline \multirow{3}{*}{ Period $t_{2}$} & $C_{1}$ & $(0.65,0.27)$ & $(0.61,0.21)$ & $(0.70,0.14)$ & $(0.44,0.37)$ & $(0.56,0.35)$ \\
\hline & $C_{2}$ & $(0.62,0.22)$ & $(0.63,0.15)$ & $(0.70,0.14)$ & $(0.70,0.20)$ & $(0.58,0.23)$ \\
\hline & $C_{3}$ & $(0.63,0.21)$ & $(0.66,0.20)$ & $(0.65,0.16)$ & $(0.77,0.19)$ & $(0.52,0.23)$ \\
\hline \multirow{3}{*}{ Period $t_{3}$} & $C_{1}$ & $(0.66,0.27)$ & $(0.66,0.24)$ & $(0.50,0.18)$ & $(0.42,0.47)$ & $(0.77,0.10)$ \\
\hline & $C_{2}$ & $(0.73,0.17)$ & $(0.75,0.16)$ & $(0.46,0.19)$ & $(0.19,0.49)$ & $(0.33,0.36)$ \\
\hline & $C_{3}$ & $(0.63,0.30)$ & $(0.73,0.20)$ & $(0.42,0.21)$ & $(0.30,0.47)$ & $(0.26,0.43)$ \\
\hline
\end{tabular}

- Step 2. The closer the evaluation value of the attribute is, the smaller the weight should be given. Otherwise, the greater weight should be given. The attribute weights for each period are calculated by Equations (22)-(24), which are shown in Table 7.

Table 7. Attribute weights in each month.

\begin{tabular}{lccc}
\hline & $w_{1}$ & $w_{2}$ & $w_{3}$ \\
\hline Period $t_{1}$ & 0.25888 & 0.29594 & 0.44518 \\
Period $t_{2}$ & 0.43661 & 0.23824 & 0.32415 \\
Period $t_{3}$ & 0.27301 & 0.37785 & 0.34914 \\
\hline
\end{tabular}

- Step 3. The comprehensive evaluation values are calculated by Equation (25) in each period, which are shown in Table 8.

Table 8. Comprehensive evaluation value by operator in each month.

\begin{tabular}{cccccc}
\hline & $A_{1}$ & $A_{2}$ & $A_{3}$ & $A_{4}$ & $A_{5}$ \\
\hline Period $t_{1}$ & $(0.599,0.322)$ & $(0.576,0.166)$ & $(0.789,0.099)$ & $(0.458,0.258)$ & $(0.404,0.342)$ \\
Period $t_{2}$ & $(0.635,0.242)$ & $(0.634,0.193)$ & $(0.683,0.146)$ & $(0.588,0.276)$ & $(0.554,0.284)$ \\
Period $t_{3}$ & $(0.675,0.244)$ & $(0.716,0.196)$ & $(0.458,0.193)$ & $(0.279,0.478)$ & $(0.383,0.324)$ \\
\hline
\end{tabular}

- Step 4. In determining the time weight, experts think the recent data is more important. The time weight vectors that are calculated by Equation (26) are $\theta(t)=(0.154,0.292,0.554)^{T}$, where $\delta=0.3$.

- Step 5. The comprehensive evaluation values are calculated by Equation (27), which are shown in Table 9.

Table 9. Comprehensive evaluation value by operator in $\delta=0.3$.

\begin{tabular}{cccc}
\hline & Comprehensive Evaluation Value by Operator & Scores Degree & Accuracy Degree \\
\hline$A_{1}$ & $(0.649,0.257)$ & 0.392 & 0.906 \\
$A_{2}$ & $(0.665,0.191)$ & 0.474 & 0.856 \\
$A_{3}$ & $(0.563,0.164)$ & 0.399 & 0.727 \\
$A_{4}$ & $(0.379,0.389)$ & -0.01 & 0.768 \\
$A_{5}$ & $(0.433,0.315)$ & 0.118 & 0.748 \\
\hline
\end{tabular}


- Step 6. The predict comprehensive evaluation values are calculated by Equations (28)-(30), which are shown in Table 10.

Table 10. Predict comprehensive evaluation value.

\begin{tabular}{cccccc}
\hline & $\boldsymbol{a}$ & $\boldsymbol{b}$ & Predict Comprehensive Evaluation Value & Scores Degree & Accuracy Degree \\
\hline$A_{1}$ & -0.09 & 0.35 & $(0.674,0.204)$ & 0.470 & 0.878 \\
$A_{2}$ & -0.17 & 0.33 & $(0.679,0.064)$ & 0.615 & 0.743 \\
$A_{3}$ & 0.68 & 1.19 & $(0.392,0.258)$ & 0.134 & 0.650 \\
$A_{4}$ & 9.04 & 3.53 & $(0.358,0.358)$ & 0.000 & 0.716 \\
$A_{5}$ & 1.28 & 0.52 & $(0.363,0.344)$ & 0.019 & 0.707 \\
\hline
\end{tabular}

- Step 7. The comprehensive evaluation values are calculated by Equation (31), which are shown in Table 11. Let $\psi=0.3$.

Table 11. Comprehensive evaluation value in $\delta=0.3$.

\begin{tabular}{cccc}
\hline & Comprehensive Evaluation Value & Scores Degree & Accuracy Degree \\
\hline$A_{1}$ & $(0.657,0.240)$ & 0.417 & 0.896 \\
$A_{2}$ & $(0.669,0.138)$ & 0.532 & 0.807 \\
$A_{3}$ & $(0.517,0.188)$ & 0.330 & 0.705 \\
$A_{4}$ & $(0.373,0.379)$ & -0.007 & 0.752 \\
$A_{5}$ & $(0.413,0.323)$ & 0.089 & 0.736 \\
\hline
\end{tabular}

All alternatives are sorted according to the ranking method of IFVs. When $\delta=0.3$, the final sort result is $A_{2} \succ A_{1} \succ A_{3} \succ A_{5} \succ A_{4}$.

- $\quad$ Step 8. Result Analysis

Result Analysis

$\delta=0.3$ shows that experts pay more attention to recent data and $\psi=0.3$ shows that experts are hesitant about future forecast data. The result of aggregation by operators is $A_{2} \succ A_{3} \succ A_{1} \succ A_{5} \succ A_{4}$, and the prediction result is $A_{2} \succ A_{1} \succ A_{3} \succ A_{5} \succ A_{4}$. This shows that, as time goes on, experts are better at evaluating $A_{1}$ than $A_{3}$. This means that $A_{1}$ is a growth candidate and $A_{3}$ is a negative candidate. Thus, companies should choose $A_{1}$ instead of $A_{3}$. This also shows that $A_{2}, A_{4}$ and $A_{5}$ are stable candidates. This means that performance of candidate $A_{2}$ is not only the best but also the most stable. Performances of $A_{4}$ and $A_{5}$ have been poor and do not recommend admission. The above analysis results are consistent with the comprehensive evaluation results obtained by future adjustment coefficients. The final result is $A_{2} \succ A_{1} \succ A_{3} \succ A_{5} \succ A_{4}$.

\section{Comparative Analysis}

(1) The proposed decision method in this paper was applied to the Case of [34], where $\delta=0.3$ and $\psi=0.3$. The result is as shown in Table 12:

Table 12. The final sort results of the case in [34].

\begin{tabular}{cll}
\hline Decision Method & Weight & Result \\
\hline Decision method in [34] & Weights used in [34] & $A_{2} \succ A_{3} \succ A_{1} \succ A_{4}$ \\
the proposed DIFPGWA operator & Weights used in [34] & $A_{2} \succ A_{3} \succ A_{1} \succ A_{4}$ \\
The TFVs-GM(1,1)-PM & Weights used in [34] & $A_{2} \succ A_{1} \succ A_{3} \succ A_{4}$ \\
Comprehensive evaluation results & Weights used in [34] & $A_{2} \succ A_{3} \succ A_{1} \succ A_{4}$ \\
the proposed DIFPGWA operator & Weights are unknown & $A_{2} \succ A_{3} \succ A_{4} \succ A_{1}$ \\
The TFVs-GM(1,1)-PM & Weights are unknown & $A_{2} \succ A_{1} \succ A_{4} \succ A_{3}$ \\
Comprehensive evaluation results & Weights are unknown & $A_{2} \succ A_{3} \succ A_{4} \succ A_{1}$ \\
\hline
\end{tabular}


The original result is $A_{2} \succ A_{3} \succ A_{1} \succ A_{4}$. If the weight information is unchanged, the result of using the proposed DIFPGWA operator is also $A_{2} \succ A_{3} \succ A_{1} \succ A_{4}$. The prediction result is $A_{2} \succ A_{1} \succ A_{3} \succ A_{4}$. The above results show that $A_{2}$ is not only the most stable but also the best. The performance of $A_{1}$ has been getting better, but the performance of $A_{3}$ has deteriorated. However, the comprehensive evaluation results are consistent with the original text, which shows that although the evaluation value of $A_{1}$ shows an upward trend, but it can not completely exceed $A_{3}$. If the weights are unknown, the result of using the proposed DIFPGWA operator is $A_{2} \succ A_{3} \succ A_{4} \succ A_{1}$. The prediction result is $A_{2} \succ A_{1} \succ A_{4} \succ A_{3}$. The final result is $A_{2} \succ A_{3} \succ A_{4} \succ A_{1}$. This result is slightly different from before because the weights are different. However, the same place is that $A_{2}$ is the best and most stable.

(2) The proposed decision method in this paper was applied to the case of [35], where $\delta=0.3$ and $\psi=0.3$. The result is as shown in Table 13:

Table 13. The final sort results of the case in [35].

\begin{tabular}{ccc}
\hline Decision Method & Weight & Result \\
\hline Decision method in [35] & Weights used in [35] & $A_{4} \succ A_{3} \succ A_{1} \succ A_{2}$ \\
the proposed DIFPGWA operator & Weights used in [35] & $A_{4} \succ A_{3} \succ A_{1} \succ A_{2}$ \\
The TFVs-GM(1,1)-PM & Weights used in [35] & $A_{1} \succ A_{3} \succ A_{4} \succ A_{2}$ \\
Comprehensive evaluation results & Weights used in [35] & $A_{1} \succ A_{3} \succ A_{4} \succ A_{2}$ \\
the proposed DIFPGWA operator & Weights are unknown & $A_{3} \succ A_{4} \succ A_{1} \succ A_{2}$ \\
The TFVs-GM(1,1)-PM & Weights are unknown & $A_{3} \succ A_{1} \succ A_{4} \succ A_{2}$ \\
Comprehensive evaluation results & Weights are unknown & $A_{3} \succ A_{1} \succ A_{4} \succ A_{2}$ \\
\hline
\end{tabular}

The original result is $A_{4} \succ A_{3} \succ A_{1} \succ A_{2}$. If the weight information is unchanged, the result of using the proposed DIFPGWA operator is also $A_{4} \succ A_{3} \succ A_{1} \succ A_{2}$. The prediction result is $A_{1} \succ A_{3} \succ A_{4} \succ A_{2}$. The comprehensive evaluation result is $A_{1} \succ A_{3} \succ A_{4} \succ A_{2}$. $A_{1}$ and $A_{3}$ rose the fastest, and $A_{1}$ exceeded $A_{3} . A_{4}$ changed from first to fourth. If the weights are unknown, the result of using the proposed DIFPGWA operator is $A_{3} \succ A_{4} \succ A_{1} \succ A_{2}$. The prediction result is $A_{3} \succ A_{1} \succ A_{4} \succ A_{2}$. The comprehensive evaluation result is $A_{3} \succ A_{1} \succ A_{4} \succ A_{2}$. This result is slightly different from before because the weights are different. When weights are unknown, both $A_{3}$ and $A_{1}$ perform better than $A_{4}$. To sum up, the performance of $A_{3}$ and $A_{1}$ is not only the best but has been in a rising stage.

(3) The proposed decision method in this paper was applied to the case of [36], where $\delta=0.3$ and $\psi=0.3$. The result is as shown in Table 14 .

Table 14. The final sort results of the case in [36].

\begin{tabular}{ccc}
\hline Decision Method & Weight & Result \\
\hline Decision method in [36] & Weights used in [36] & $A_{1} \succ A_{2} \succ A_{3}$ \\
the proposed DIFPGWA operator & Weights used in [36] & $A_{1} \succ A_{2} \succ A_{3}$ \\
The TFVs-GM(1,1)-PM & Weights used in [36] & $A_{2} \succ A_{1} \succ A_{3}$ \\
Comprehensive evaluation results & Weights used in [36] & $A_{2} \succ A_{1} \succ A_{3}$ \\
the proposed DIFPGWA operator & Weights are unknown & $A_{1} \succ A_{2} \succ A_{3}$ \\
The TFVs-GM(1,1)-PM & Weights are unknown & $A_{1} \succ A_{2} \succ A_{3}$ \\
Comprehensive evaluation results & Weights are unknown & $A_{1} \succ A_{2} \succ A_{3}$ \\
\hline
\end{tabular}

The original result is $A_{1} \succ A_{2} \succ A_{3}$. If the weight information is unchanged, the result of using the proposed DIFPGWA operator is $A_{1} \succ A_{2} \succ A_{3}$. The prediction result is $A_{2} \succ A_{1} \succ A_{3}$. The comprehensive evaluation result is $A_{2} \succ A_{1} \succ A_{3}$. This shows that the rise of $A_{2}$ exceeds $A_{1}$, and the problem is not considered in the original text. If the weights are unknown, the result of using the 
proposed DIFPGWA operator is $A_{1} \succ A_{2} \succ A_{3}$. The prediction result is $A_{1} \succ A_{2} \succ A_{3}$. The above results are basically the same- that $A_{1}$ is the best, $A_{2}$ is the second, and $A_{3}$ is the worst.

After the above comparative analysis, it is obvious that the decision method proposed in this paper is not only effective but also more advantageous.

\section{Conclusions}

DIF-MAGDM is an important branch of modern decision theory. Because of this, the DIFPGWA operator is proposed. The operator makes up for the shortcomings of the previous decision-making method and better reflects the support relationship between attributes, between substitutions, and between periods. The DIFPGWA operator can capture the subtle details of the comprehensive evaluation value that the decision maker needs to reflect. However, judging only by aggregating evaluation information from past to present is inaccurate. Therefore, the TFVs-GM(1,1)-PM is improved in this paper. The previous IFVs-GM-(1,1)-PM predicts that the membership and membership of IFVs may be out of range. Our improved IFVs-GM(1,1)-PM limits membership and non-affiliation within $[0,1]$. The DIFPGWA operator and the IFVs-GM(1,1)-PM complement each other. The future adjustment coefficient is proposed, which combines the two results. This method can help decision-makers to make more accurate judgments. It is very important to determine the weights in DIF-MAGDM. In this article, attribute weights, expert weights, and time weights are all unknown. This paper adopts the idea of deriving expert weight from the evaluation value and improves the previous method. At each period, therefore, it is generally agreed that the more deviated from the common cognition, the smaller the expert weight should be, and, the closer to the common cognition, the greater the expert weight should be. However, if the experts evaluate the same value, the sum of the deviations will be zero. Therefore, it will lead to no solution to the weight of experts. Our improved approach avoids this error. For attribute weights, the decision maker can determine the weight of each period's attributes based on the idea of maximum deviation. That is, the closer the evaluation value of the attribute is, the smaller the weight that should be given. Otherwise, it should be given greater weight. Moreover, due to different evaluations of experts in different periods, attribute weights should also be different. For time weights, the decision maker can use subjective and objective methods to determine the time weights. Subjective can fully consider the knowledge and experience of experts, treat things with their own feelings, and draw conclusions. Objective can make full use of all the time information. Therefore, the combination of subjectivity and objectivity can give more reasonable time weights. The decision method proposed in this paper is applied to the selection of the company's intern employees. After three months of internship, two excellent and up-scaling employees were selected. Not only that, this article also applies the proposed decision method to other cases. The final comparative analysis shows that the decision-making method is more effective and superior. In the future, we will first apply the decision method proposed in this paper to practical cases, and second apply this method to more fields.

Author Contributions: Conceptualization, K.Y.; Formal Analysis and Writing-Original Draft, P.W.; Writing-Review and Editing, X.J.

Funding: This work was supported bythe Major Program (Grant No. 14ZDB151) and State Key Program (Grant No. 16AZD018) of the National Social Science Foundation of China, the Youth Program of the National Social Science Foundation of China (Grant No. 18CJY018); the Ministry of Education Philosophy and Social Sciences Development Report Breeding Project (Grant No. 13JBGP005); the National Key Research and Development Program of China (Grant No. 2016YFC1402000); the National Natural Science Foundation of China (Grant No. 41701593, No. 71371098, No. 71571157); the National Special Science Foundation for Postdoctoral Scientists of China (Grant No. 2018T110708); the National Science Foundation for Postdoctoral Scientists of China (Grant No. 2015M580611, 2017M610446); the Social Science Foundation of Shandong Province (Grant No. 17DJJJ07) and Qingdao City, China (Grant No. QDSKL1701010); the Science Foundation for Postdoctoral Scientists of Qingdao City, China (Grant No. 24, 251); the National Special Research Fund for Non-Profit Marine Sector (Grant No. 201305034, No. 201405029); and the Fundamental Research Funds for the Central Universities (201613006, 201564031). 
Acknowledgments: The authors would like to thank the editors and the anonymous reviewers for their constructive comments and suggestions, which have helped to improve the paper.

Conflicts of Interest: The authors declare no conflict of interest

\section{References}

1. Atanassov, K.T. Intuitionistic fuzzy sets. Fuzzy Sets Syst. 1986, 20, 87-96. [CrossRef]

2. Bao, T.T.; Xie, X.L.; Meng, P.P. Intuitionistic fuzzy hybrid multi-criteria decision making based on prospect theory and evidential reasoning. Syst. Eng. Theory Pract. 2017, 37, 460-468.

3. Chen, Q.W. Application of Intuitionistic Fuzzy Entropy Weight Method to Evaluate Quality of Medical Care. J. Math. Med. 2010, 23, 85-486.

4. Yu, G.F.; Li, D.F.; Qiu, J.M. Solution to intuitionistic fuzzy linear programming and its application. Control Decis. 2015, 30, 640-644.

5. Li, B.P.; Chen, H.Y. Intuitionistic Fuzzy Area Set and Its Application in Pattern Recognition. Fuzzy Syst. Math. 2015, 29, 134-141.

6. Liu, P.D.; Li, Y.; Antuchevičienè, J. Multi-criteria decision-making method based on intuitionistic trapezoidal fuzzy prioritised OWA operatorm. Technol. Econ. Dev. Econ. 2016, 22, 453-469. [CrossRef]

7. Hashemi, H.; Mousavi, S.M.; Zavadskas, E.K.; Chalekaee, A.; Turskis, Z. A New Group Decision Model Based on Grey-Intuitionistic Fuzzy-ELECTRE and VIKOR for Contractor Assessment Problem. Sustainability 2018, 10, 1635. [CrossRef]

8. Kahraman, C.; Ghorabaee, M.; Zavadskas, E.; Onar, S.; Yazdani, M.; Oztaysi, B. Intuitionistic fuzzy EDAS method: An application to solid waste disposal site selection. J. Environ. Eng. Landsc. Manag. 2017, $25,1-12$. [CrossRef]

9. Yager, R.R. OWA aggregation of intuitionistic fuzzy sets. Int. J. Gen. Syst. 2009, 38, 617-641. [CrossRef]

10. Xu, Z.S. Intuitionistic Fuzzy Aggregation Operators. IEEE Trans. Fuzzy Syst. 2007, 15, 1179-1187.

11. Xu, Z.S.; Yager, R.R. Some geometric aggregation operators based on intuitionistic fuzzy sets. Int. J. Gen. Syst. 2006, 35, 417-433. [CrossRef]

12. Tan, C.Q.; Chen, X.H. Intuitionistic fuzzy Choquet integral operator for multi-criteria decision making. Int. J. Intell. Syst. 2011, 26, 659-686. [CrossRef]

13. Xu, Z.S. Approaches to Multiple Attribute Group Decision Making Based on Intuitionistic Fuzzy Power Aggregation Operators; Elsevier Science Publishers B. V.: New York, NY, USA, 2011.

14. Wei, G.W. Gray Relational Analysis Method for Intuitionistic Fuzzy Multiple Attribute Decision Making. Expert Syst. Appl. 2011, 38, 11671-11677. [CrossRef]

15. Yue, Z.L. TOPSIS-based group decision-making methodology in intuitionistic fuzzy setting. Inf. Sci. 2014, 277, 141-153. [CrossRef]

16. Li, P.; Wu, J.M.; Zhu, J.J. Stochastic multi-criteria decision-making methods based on new intuitionistic fuzzy distance. Syst. Eng. Theory Pract. 2014, 34, 1517-1524.

17. Dymova, L.; Sevastjanov, P. A new approach to the rule-base evidential reasoning in the intuitionistic fuzzy setting. Knowl-Based Syst. 2014, 61, 109-117. [CrossRef]

18. Iancu, I. Intuitionistic fuzzy similarity measures based on Frank t-norms family. Pattern Recognit. Lett. 2014, 42, 128-136. [CrossRef]

19. Xu, Z.S.; Yager, R.R. Dynamic intuitionistic fuzzy multi-attribute decision making. Int. J. Approx. Reason. 2008, 48, 246-262. [CrossRef]

20. Wei, G.W. Some geometric aggregation functions and their application to dynamic multiple attribute decision making in the intuitionistic fuzzy setting. Int. J. Uncertain. Fuzziness Knowl-Based Syst. 2009, 17, 179-196. [CrossRef]

21. Su, Z.X.; Chen, M.Y.; Xia, G.P.; Wang, L. An interactive method for dynamic intuitionistic fuzzy multi-attribute group decision making. Expert Syst. Appl. 2011, 38, 15286-15295. [CrossRef]

22. Park, J.H.; Cho, H.J.; Kwun, Y.C. Extension of the VIKOR method to dynamic intuitionistic fuzzy multiple attribute decision making. Comput. Math. Appl. 2013, 65, 731-744. [CrossRef]

23. Chen, W.; Yang, Z.L.; Zhou, W.; Chen, H. Dynamic Intuitionistic Fuzzy Compromise Decision Making Method Based on Time Degrees. Oper. Res. Manag. Sci. 2016, 25, 83-89.

24. Li, P.; Liu, S.F.; Zhu, J.J. GM(1,1) PM based on intuitionistic fuzzy numbers. Control Decis. 2013, 28, 1583-1586. 
25. Chen, Z.P.; Yang, W. A new multiple attribute group decision making method in intuitionistic fuzzy setting. Appl. Math. Model. 2011, 35, 4424-4437. [CrossRef]

26. Wang, Y.M. Using the method of maximizing deviation to make decision for multiindices. J. Syst. Eng. Electron. 1997, 8, 21-26.

27. Wu, Z.B.; Chen, Y. The Maximizing Deviation Method for Group Multiple Attribute Decision Making under Linguistic Environment; Elsevier North-Holland, Inc.: New York, NY, USA, 2007.

28. Guo, Y.J.; Yao, Y.; Yi, P.T. A Method and Application of Dynamic Comprehensive Evaluation. Syst. Eng. Theory Pract. 2007, 27, 154-158. [CrossRef]

29. Xu, Z.S. Intuitionistic preference relations and their application in group decision making. Inf. Sci. Int. J. 2007, 177, 2363-2379. [CrossRef]

30. Chen, S.M.; Tan, J.M. Handling Multicriteria Fuzzy Decision-Making Problems Based on Vague Set Theory; Elsevier North-Holland, Inc.: New York, NY, USA, 1994.

31. Hong, D.H.; Choi, C.H. Multicriteria fuzzy decision-making problems based on vague set theory. Fuzzy Sets Syst. 2000, 114, 103-113. [CrossRef]

32. Aczél, J.; Saaty, T.L. Procedures for synthesizing ratio judgements. J. Math. Psychol. 1983, 27, 93-102. [CrossRef]

33. Xu, Z.S.; Yager, R.R. Power-Geometric Operators and Their Use in Group Decision Making. IEEE Trans. Fuzzy Syst. 2010, 18, 94-105.

34. Zhang, Y.Y.; Feng, Q.; Zhou, D.Y.; Zhang, K. Multi-Attribute Dynamic Threat Assessment in Air Combat Based on Intuitionistic Fuzzy Sets. Electron. Opt. Control 2015, 22, 17-21.

35. Mei, X.L. Dynamic Intuitionistic Fuzzy Multi-attribute Decision Making Method Based on Similarity. Stat. Decis. 2016, 15, 22-24.

36. Du, Y.; Wu, Z.Q.; Li, D.F. Dynamic Intuitional Fuzzy Multiple Attribute Group Decision-making Method Based on DIFHA Operator. Fire Control Command Control 2011, 8, 15-18.

(C) 2018 by the authors. Licensee MDPI, Basel, Switzerland. This article is an open access article distributed under the terms and conditions of the Creative Commons Attribution (CC BY) license (http://creativecommons.org/licenses/by/4.0/). 